Imperial-TP-93-94-44

hep-th/9406182

\title{
Quantum Backreaction on "Classical" Variables
}

\author{
Arlen Anderson*广 \\ Isaac Newton Institute \\ 20 Clarkson Road \\ Cambridge CB3 0EH, England \\ and \\ Blackett Laboratory \\ Imperial College \\ Prince Consort Rd. \\ London SW7 2BZ, England
}

June 26, 1994

\begin{abstract}
A mathematically consistent procedure for coupling quasiclassical and quantum variables through coupled Hamilton-Heisenberg equations of motion is derived from a variational principle. During evolution, the quasiclassical variables become entangled with the quantum variables with the result that the value of the quasiclassical variables depends on the quantum state. This provides a formalism to compute the backreaction of any quantum system on a quasiclassical one. In particular, it leads to a natural candidate for a theory of gravity coupled to quantized matter in which the gravitational field is not quantized.
\end{abstract}

*arley@physics.unc.edu

†Present address: Dept. Physics, UNC-Chapel Hill, Chapel Hill NC 27599-3255. 
Few would argue with the view that the universe is wholly quantum, and that classical physics is only an approximate description of a collection of phenomena that emerge in certain situations 11, 2]. This view has not always been universally held, especially concerning the quantization of the gravitational field[3]. In this Letter, working within the simplified context of quantum mechanics, I shall present and interpret a mathematically consistent scheme for coupling "classical" and quantum variables. This has a dual purpose. It is intended to assuage mathematical doubts about how classical and quantum variables may coexist in a single theory. Secondly, this approach serves as an approximate description of any fully quantum mechanical theory when some of the variables behave essentially classically. It provides a means of computing the backreaction that quantum variables have on the evolution of classical ones without having to make a full semiclassical analysis.

Confidently assuming that the same scheme extends to field theory, this approach has potentially far-reaching implications. By presenting the outline of a new theory in which the gravitational field is not quantized, it reopens the debate on the necessity of quantizing gravity[3, 4]. Alternatively, when viewed as an approximation to a fully quantum theory of gravity in energy regimes where quantum gravitational effects can be ignored, it improves on conventional quantum field theory in curved spacetime by taking account of the backreaction of quantum matter fields on the classical spacetime background. The effects of backreaction are expected to be very important for a complete understanding of information loss in black hole evaporation [5]. It is an interesting question whether the predictions of a fully quantum theory of gravity would differ in an experimentally detectable way from those of this type of formulation.

The approach followed here is similar in motivation to one discussed independently by Aleksandrov [6] and by Boucher and Traschen [7], but it differs in details and has an entirely original derivation and interpretation. The key feature is that the classical variables evolve to become correlated with the state of the quantum variables. Because this correlation may be with different states in a quantum superposition, the classical variables need not have a definite value but may take a distribution of values depending on the quantum state. This parallels the behavior one expects in a fully quantum system as some variables become classical (cf. [2]). These variables are not then entirely classical, but may be more properly called quasiclassical.

This behavior stands in contrast to that predicted by the traditional ap- 
proach of coupling classical variables to the expectation value of quantum ones 3, 4. As emphasized by Kibble [4, the traditional approach amounts to a nonlinear modification of quantum theory. The quasiclassical approach takes the easier route of accepting linear quantum mechanics yet allowing the value of the quasiclassical variables to depend on the quantum state. By relaxing the requirement that quasiclassical variables take a single definite value, a state-dependent backreaction of the quantum variables on the quasiclassical evolution is made possible. Effectively the quantum variables act like a source of noise on the quasiclassical evolution. (In this connection, the approach of $\mathrm{Hu}\|8\|$ to include stochastic corrections to traditional semiclassical gravity should be mentioned as a possibly related alternative.) One might ask in what sense the quasiclassical variables are classical if it is not because they take definite values. The answer is that they are classical because they do not exhibit self-interference effects. That is, in the absence of coupling to a quantum system, the quasiclassical variables evolve classically without interference phenomena.

To elaborate on this, consider a fully quantum system in which some variables evolve essentially classically, that is, as well localized wave packets with minimal dispersion, when coupling to the remaining variables is neglected. In the presence of coupling, quantum fluctuations of these other variables can lead the "classical evolution" to divide into a collection of qualitatively distinct evolutions, most easily by triggering instabilities. By appealing to the concept of decoherence [1, 2, 9], one finds that each of these evolutions occurs with a classical probability as the overlap between states associated to different evolutions tends to zero. This phenomenon in which the distribution among possible quasiclassical evolutions is controlled by quantum fluctuations in the non-quasiclassical variables is potentially important in many areas of physics where the classical and quantum regimes overlap. It may be particularly important in the early universe. In a different context, quantum fluctuations of a scalar field amplified by inflation have already been proposed as the source of fluctuations in the cosmic microwave background radiation and as seeds for galaxy formation [10].

The first step in the proposal for coupling quasiclassical and quantum variables is to characterize each type of variable. Viewed algebraically, quantum variables are noncommutative variables satisfying commutation relations. To be explicit, consider one variable and its conjugate with the canonical commutation relation $[q, p]=i(\hbar=1)$. One can define a non- 
commutative algebra $\mathcal{U}^{(q)}$ of functions of $q, p$ involving arbitrary complex powers of each. This is essentially the algebra of pseudodifferential operators, and it contains all observables, as well as non-self-adjoint elements. Quantum canonical transformations are maps between canonically conjugate pairs of elements which preserve the canonical commutation relations 11, 12]. They are generated by the adjoint action of an element, say, $C \in \mathcal{U}^{(q)}$, $C:(q, p) \mapsto\left(C q C^{-1}, C p C^{-1}\right)$.

The Hamiltonian $H(q, p, t)$ at a fixed time is an element of $\mathcal{U}^{(q)}$, and it can be used to define a quantum action,

$$
S=\int_{t_{1}}^{t_{2}} d t \frac{1}{2}(p \dot{q}+\dot{q} p)-H(q, p, t),
$$

where the integral is over a path in $\mathcal{U}^{(q)}$. The Heisenberg equations of motion can be derived by a quantum variational principle. Variations are defined in terms of the infinitesimal canonical transformations which generate translations of $q$ and $p$. Requiring that the action be stationary under such variations with fixed endpoints leads to the Heisenberg equations of motion[13]. A canonical transformation is simply a change of basis in $\mathcal{U}^{(q)}$, and the Heisenberg equations of motion are invariant under them. For a time-independent Hamiltonian, evolution in the Heisenberg picture is produced by the unitary canonical transformation $C=e^{i H t}$.

(Quasi)classical variables satisfy Poisson bracket relations. Again, consider the case of one variable with $\{x, k\}=1\left(\{f, g\}=\partial_{x} f \partial_{k} g-\partial_{k} f \partial_{x} g\right)$. Classical mechanics can be formulated by working from a commutative algebra of functions $\mathcal{U}^{(c)}$ of the phase space variables. Canonical transformations are maps between canonically conjugate pairs of elements of this algebra. The action can be defined as an integral over a path in $\mathcal{U}^{(c)}$ as in (1). In parallel to the quantum case, the Hamilton equations of motion are obtained by requiring that the action is stationary under variations generated by the infinitesimal canonical transformations which generate translations in $x$ and $k$. For a time-independent Hamiltonian, evolution is produced by the unitary transformation $C=e^{v_{H} t}$, where $v_{H} f=\partial_{x} f \partial_{k} H-\partial_{k} f \partial_{x} H$ defines the action of the Hamiltonian vector field $v_{H}$ generated by $H$ on a function $f \in \mathcal{U}^{(c)}$.

Given this strongly parallel treatment of the equations of motion for quantum and (quasi)classical variables, it is natural to couple them by working with an algebra $\mathcal{U}^{(q-c)}$ of functions of both commutative elements $x, k$ satisfying the Poisson bracket relation $\{x, k\}=1$ and noncommutative elements 
$q, p$ satisfying the canonical commutation relation $[q, p]=i$. All other brackets between these elements are assumed to vanish. (Note that $x, k, q, p$ are essentially generators of the algebra $\mathcal{U}^{(q-c)}$ and should be distinguished from the dynamical variables $x(t), k(t)$, etc. which happen to take those specific values at the initial time.) Two general elements $A, B$ of $\mathcal{U}^{(q-c)}$, involving both commutative and noncommutative variables, are canonically conjugate with respect to a quasiclassical bracket if they satisfy the relation

$$
[A, B]_{(q-c)}=[A, B]+i\{A, B\}=i
$$

The order of quantum variables is preserved when evaluating the Poisson bracket: thus, if $U, V$ are functions of the quantum variables and $f, g$ are functions of the quasiclassical variables, one has the quasiclassical bracket $[f U, g V]_{(q-c)}=f g[U, V]+i U V\{f, g\}$. This quasiclassical bracket differs from the definition in Refs. [6] and [0] and in particular is not antisymmetric.

Since every element of $\mathcal{U}^{(q-c)}$ is expressed in terms of $q, p, x$ and $k$, canonical transformations can be characterized by their action on these elements. Elementary canonical transformations 11] can be defined which transform the noncommutative elements $q, p$ as quantum variables and the commutative ones $x, k$ as classical variables - that is, without sensing the mixed character of the quasiclassical bracket. A large class of (essentially all useful) canonical transformations are produced by composition of elementary transformations. In particular composition produces transformations which are of mixed character, and one finds the bracket which is preserved under composition is the quasiclassical bracket above. This justifies the choice of this bracket. A general canonical transformation is then a map between pairs of canonically conjugate elements, which preserves the quasiclassical bracket relations $[A, B]_{(q-c)}=i$. Note that if one has $[f, g]=0$ or $\{U, V\}=0$ initially, these relations are not generically preserved by general canonical transformations.

For a Hamiltonian $H(q, p, x, k, t)$, a variational principle analogous to those above shows that the coordinates $(q(t), p(t), x(t), k(t))$ satisfy coupled Hamilton-Heisenberg equations of motion, which at the initial time read

$$
\begin{aligned}
\dot{q}=-i[q, H] \quad, \quad \dot{p}=-i[p, H], \\
\dot{x}=\{x, H\} \quad, \quad \dot{k}=\{k, H\} .
\end{aligned}
$$

A function $A(q, p, x, k, t)$ of both quasiclassical and quantum variables satis- 
fies

$$
\frac{d A}{d t}=-i[A, H]_{(q-c)}+\frac{\partial A}{\partial t}
$$

If $d H / d t=0$, evolution in the "Heisenberg picture" is generated by $e^{v_{H}^{(q-c)} t}$, where $v_{H}^{(q-c)}$ is the quasiclassical analog of a Hamiltonian vector field $v_{H}^{(q-c)} f=-i[f, H]_{(q-c)}$. Since the quasiclassical bracket is not antisymmetric, it is possible for a time-independent Hamiltonian to have nontrivial evolution

$$
d H / d t=-i[H, H]_{(q-c)} \neq 0 .
$$

This is an unusual feature of this proposal, apparently related to factor ordering of the Hamiltonian as the coordinates evolve. Evolution in this case is not yet well understood [14].

The coupled Hamilton-Heisenberg equations of motion are invariant under general canonical transformations which mix the quantum and quasiclassical degrees of freedom, so their solutions can be computed in any basis. An S-matrix computed in one basis is equivalent to one computed in another, and this answers Duff's objection[15] that such transformations would not preserve the S-matrix in a theory with both classical and quantum variables 16.

It is clear from the equations of motion (3) that the quasiclassical variables will evolve to depend on the quantum variables $q, p$, and vice versa. The latter condition is familiar: the quasiclassical variables can be viewed simply as c-number parameters in the Heisenberg operators $q(t)$ and $p(t)$. That the quasiclassical variables come to depend on $q$ and $p$ is the distinguishing feature of this proposal and leads to state-dependent values for $x(t)$ and $k(t)$. Choose as initial state the product of a quantum state (possibly a density matrix) and a pair of definite values for the quasiclassical variables (or a density matrix describing their positive joint probability distribution). In the Heisenberg picture, one can compute expectation values of observables at time $t$ in the usual way $-x$ and $k$ act as multiplication operators on the initial state, taking the values of the initial quasiclassical positions and momenta (with their respective probabilities).

Alternatively, suppose that $x(t)=A(q, p, x, k, t)$. The operator dependence may be interpreted by decomposing the initial quantum state into eigenstates of $A$, using the initial quasiclassical values for $x$ and $k$. Then, the probability that $x(t)$ takes a given value $\lambda$ is the probability that the quantum state is in the eigenstate having the eigenvalue $\lambda$. 
One may well ask whether the joint distribution of values for $(x(t), k(t))$ is necessarily a positive probability distribution. The answer is that it is not. This should not be unexpected. If evolution leads to $[x(t), k(t)] \neq 0$, as it may, $x(t)$ and $k(t)$ cannot be simultaneously measured, and they won't necessarily have a positive joint probability distribution. A consequence is that the Schrödinger representation can be problematic because the evolved quasiclassical system is not obviously described by a state (or even a density matrix). This is a topic for further study.

One might argue that the quasiclassical variables are no longer very classical because they have lost the property of simultaneous measurability. The essential point though is that this has arisen because of their entanglement with the quantum variables and not because of intrinsic self-interference. One expects that a coarse-graining on the scale of the quantum variables will render the joint distribution positive. Incidentally, it is the fact that $x(t)$ and $k(t)$ are limited in their simultaneous measurability by one's ability to put the quantum system in a simultaneous eigenstate of each that prevents one from violating the uncertainty principle by making measurements of the quasiclassical variables.

An example will illustrate the proposal. Consider the Hamiltonian $H=$ $\frac{1}{2} k p^{2}$. The solutions are easily found to be $p(t)=p, k(t)=k, q(t)=q+k p t$, $x(t)=x+\frac{1}{2} p^{2} t$. Since $x(t)$ and $k(t)$ commute with $H$, one can work in the Schrödinger picture by decomposing the initial state into eigenfunctions of $H$ : the wavefunction of the quantum particle is expanded in plane waves and the initial conditions of the quasiclassical particle are labeled $\left|\left(x^{\prime}, k^{\prime}\right)\right\rangle$. The propagator can be formally denoted $e^{-i H t}$ which is understood to give the usual phase factor involving the eigenvalue of $H$ when it acts on the initial state. It also evolves the quasiclassical variables to their final operator values which then act on the quantum state. (Generally Schrödinger picture evolution is more subtle than this and requires discussion beyond the scope of this Letter.) The evolved state of the full system is

$$
\begin{aligned}
\Psi & =e^{-i H t} \int d p^{\prime} f\left(p^{\prime}\right) e^{i p^{\prime} q}\left|\left(x^{\prime}, k^{\prime}\right)\right\rangle \\
& =\int d p^{\prime} f\left(p^{\prime}\right) e^{i p^{\prime} q} e^{-\frac{i}{2} k^{\prime} p^{\prime 2} t}\left|\left(x^{\prime}+\frac{1}{2} p^{2} t, k^{\prime}\right)\right\rangle .
\end{aligned}
$$

If the wavepacket for the quantum particle is highly concentrated around the momenta $p_{1}^{\prime}$ and $p_{2}^{\prime}$ with amplitudes $\psi_{1}(q)$ and $\psi_{2}(q)$ respectively, then the 
state of the full system is approximately

$$
\Psi \approx \psi_{1}(q)\left|\left(x^{\prime}+\frac{1}{2} p_{1}^{\prime 2} t, k^{\prime}\right)\right\rangle+\psi_{2}(q)\left|\left(x^{\prime}+\frac{1}{2} p_{2}^{\prime 2} t, k^{\prime}\right)\right\rangle .
$$

The probability that the classical particle is at $x(t)=x^{\prime}+\frac{1}{2} p_{1}^{\prime 2} t$ at time $t$ is $\int\left|\psi_{1}(q)\right|^{2} d q$.

A second example shows three more features of the coupling of quasiclassical and quantum variables. Consider the Hamiltonian

$$
H=\frac{1}{2} p^{2}+a k q
$$

The solution to the equations of motion are $k(t)=k, p(t)=p-a k t, q(t)=$ $q+p t-\frac{1}{2} a k t^{2}$ and

$$
x(t)=x+a \int_{0}^{t} d t q(t)=x+a q t+\frac{1}{2} a p t^{2}-\frac{1}{6} a^{2} k t^{3} .
$$

The first thing to note is that $x(t)$ does not depend on the instantaneous eigenvalue of a fixed operator but has an accumulated dependence over time. Thus the operator which determines the distribution of values of $x(t)$ changes dynamically.

Next, there is a feedback term $-a^{2} k t^{3} / 6$ present. This arises because the quasiclassical variables influence the quantum variables which in turn act back on the quasiclassical variables. The result is a quasiclassical term in the evolution which would not be present except through the quantum coupling. It is a second order effect as one expects.

The solution to the Hamilton-Heisenberg equations of motion are the Heisenberg picture operators. This means that it is easy to compute any expectation value at a later time in terms of expectation values at the initial times. In particular, the variance of $x(t)$ is

$$
\Delta x(t)=\Delta\left(q+\frac{1}{2} p t\right) a t .
$$

This shows that while one can squeeze the initial quantum state to make the variance in $x(t)$ vanish at a particular later instant, the variance cannot be held small.

Since the quantum state does not have a definite value, in equations of motion like $\dot{x}=q$, the quantum variable behaves as a noise term. This raises 
the intriguing possibility of using quasiclassical quantum theory as a way of solving classical stochastic differential equations. It is clear that one is solving a stochastic differential equation by this approach. The question of whether one can solve stochastic differential equations which are of interest is under investigation.

The proposal for a quasiclassical theory of gravity is to solve the coupled equations

$$
G_{\mu \nu}=8 \pi T_{\mu \nu}, \quad T_{; \nu}^{\mu \nu}=0,
$$

where the metric is assumed to be quasiclassical and the matter fields are quantum. There are several difficulties to be overcome in implementing this in practice. The most obvious is simply finding any solutions to the equations. As a first step, a covariant gauge-invariant perturbative approach 17 seems natural. In the larger picture, there are questions about the nature of the Hilbert space for the quantum variables as this depends on the choice of background in a way that the Hilbert space in the quantum mechanical context did not depend on the quasiclassical variables. This is but one aspect of the problem of time 18, involving the definition of quantum theory in curved backgrounds, which must be addressed.

Acknowledgements. I would like to thank the participants, especially R. LaFlamme, of the program of Geometry and Gravity at the Newton Institute for helpful discussions of this work.

\section{References}

[1] J.B. Hartle, in Gravitation and Quantizations, Proceedings of the 1992 Les Houches Summer School, vol. LVII, North-Holland, Amsterdam (1994).

[2] M. Gell-Mann and J.B. Hartle, Phys. Rev. D 47, 3345 (1993).

[3] Cf. critical remarks by L. Rosenfeld, Nucl. Phys. 40, 353 (1963).

[4] T.W.B. Kibble, in Quantum Gravity 2, edited by C.J. Isham, R. Penrose and D.W. Sciama, (Clarendon Press, Oxford, 1981), 63.

[5] For a review with comprehensive bibliography, see D. Page, Proceedings of the 5th Canadian Conference on General Relativity and Relativistic 
Astrophysics, edited by R.B. Mann and R.G. McLenaghan (World Scientific, Singapore, 1994).

[6] I.V. Aleksandrov, Z. Naturf. 36A, 902 (1981).

[7] W. Boucher and J. Traschen, Phys. Rev. D37, 3522 (1988).

[8] B.L. Hu and S. Sinha, preprint Univ. Maryland 93-164 (1993); B.L. Hu, preprint Univ. Maryland 94-45, gr-qc/940361 (1994).

[9] W. Zurek, Physics Today 44 (10), 36 (1993); in Physical Origins of Time Asymmetry, edited by J.J. Halliwell, J. Pérez-Mercader, and W.H. Zurek, (Cambridge Univ. Press, Cambridge, 1994), 175-207.

[10] A. Guth and S.Y. Pi, Phys. Rev. Lett. 49, 1110 (1982); S. Hawking, Phys. Lett. B 115, 295 (1982); A. Starobinskii, Phys. Lett. B 117, 175 (1982).

[11] A. Anderson, Ann. Phys. (NY) 232, 292 (1994); Phys. Lett. B 319, 157 (1993).

[12] M. Born, W. Heisenberg, and P. Jordan, Ztschr. f. Phys. 35, S. 557 (1926); P.A.M. Dirac, Proc. Roy. Soc. A110, 561 (1926).

[13] J. Schwinger, Quantum Kinematics and Dynamics, (Benjamin: New York, 1970); A. Anderson, in preparation.

[14] L.L. Salcedo, private communication.

[15] M.J. Duff, in Quantum Gravity 2, edited by C.J. Isham, R. Penrose and D.W. Sciama, (Clarendon Press, Oxford, 1981), 81.

[16] Other aspects of Duff's argument, in particular, the invariance of renormalization under canonical transformations, need further attention in field theory.

[17] G.F.R. Ellis and M. Bruni, Phys. Rev. D40, 1804 (1989).

[18] K. Kuchar in Proceedings of the 4th Canadian Conference on General Relativity and Astrophysics, edited by G. Kunstatter, D. Vincent, and J. Williams (World Scientific, Singapore, 1992). 